Original Paper http://ajol.info/index.php/ijbcs http://indexmedicus.afro.who.int

\title{
Données préliminaires sur l'exploitation des pangolins (Pholidota) autour du Parc National de Kahuzi-Biega, République Démocratique du Congo
}

\author{
J. Mapoli MBUSA ${ }^{1,2 *}$ J. Nyumu KAMBALE ${ }^{2,3}$, D. Malimbo KAMBALE ${ }^{1,2}$, \\ B. Visando KAMBALE ${ }^{1,2}$, Francis Nchembi TARLA ${ }^{4}$, J.C. Itoka MUKINZI ${ }^{5}$, \\ S. Mbalitini GAMBALEMOKE ${ }^{5}$
}

\author{
${ }^{1}$ Département de Zooécologie, Université de Conservation de la Nature et de Développement de Kasugho, \\ République Démocratique du Congo $(R D C)$. \\ ${ }^{2}$ Research Center for Environmental Planning (RCEP-Goma/RDC). \\ 3 Doctorant à l'Université de Kisangani (RDC). \\ ${ }^{4}$ Central Africa BushMeat Action Group $(C A B A G)$ et US Fish Wildlife, République du Cameroun. \\ 5 Professeurs à l'Université de Kisangani (RDC). \\ *Auteur correspondant ; E-mail :jmapoli5@gmail.com
}

\section{REMERCIEMENTS}

Les auteurs tiennent à remercier tous ceux qui, d'une manière ou d'une autre, ont contribué à la réalisation de ce travail. Ces remerciements vont particulièrement à l'endroit des relecteurs anonymes du manuscrit ainsi qu'à toute personne ayant accepté de répondre au questionnaire proposé, pour leur disponibilité et amabilité.

\section{RESUME}

Dans un contexte où la demande des écailles des pangolins pour la médicine traditionnelle chinoise ne cesse d'augmenter, ces animaux se trouvent de plus à plus braconner. Il est donc important que les études soient menées dans leur zone de répartition pour arriver à dégager les motivations de cette exploitation dans chaque zone. C'est dans cet angle que l'étude sur l'exploitation des pangolins et de leurs produits dérivés a été réalisée autour du parc national de Kahuzi-Biega avec pour objectif de dégager les différentes fins pour lesquelles ces animaux y sont exploités et identifier les différents acteurs intervenant dans ce domaine via l'information et les connaissances des riverains. La méthode d'enquête individuelle et collective a été conduite auprès de 116 personnes pendant une période de deux mois (mars et avril 2019) autour du Secteur Tshivanga et du Secteur Itebero. Les analyses comparatives, Khi-deux, Ficher et les analyses à correspondances multiples $(\mathrm{ACM})$ ont été effectuées à l'aide du logiciel Past 3.2. Les résultats obtenus montrent que les pangolins sont principalement exploités pour l'alimentation et la médecine traditionnelles. Cependant, le trafic de leurs écailles a fait augmenter la demande dans la zone. Les demandeurs des écailles viennent principalement des grandes villes (Bukavu, Goma, Kisangani) et des certaines pays étrangers (Ouganda, Burundi, Tanzanie et les agents de la Mission des Nations-Unies pour la Stabilisation au Congo). Le commerce des écailles pour satisfaire la demande externe, bien qu'encore à ces débuts, vient menacer les pangolins dans cette zone où la chasse est strictement interdite. Ce commerce passe par des intermédiaires, dont les principaux sont les enfants du milieu.

(C) 2020 International Formulae Group. All rights reserved.

Mots clés : Étude préliminaire, exploitation, pangolins, Kahuzi-biega, RDC 


\title{
Preliminary data on the exploitation of Pangolins (Pholidota) about the National Park of Kahuzi-Biega, Democratic Republic of Congo (DRC)
}

\begin{abstract}
In a context where the demand for pangolin scales for traditional Chinese medicine continues to increase, these animals are increasingly being poached. It is therefore important that studies be carried out in their area of distribution in order to identify the motivations for this exploitation in each area. It is in this perspective that the study on the exploitation of pangolins and their by-products was carried out around the Kahuzi-Biega National Park with the aim of identifying the different purposes for which these animals are exploited there and to identify the different actors involved in this field through the information and knowledge of local residents. The individual and collective survey method was conducted among 116 people over a twomonth period (March and April 2019) around the Tshivanga Sector and the Itebero Sector. Comparative, Chisquare, File and Multiple Correspondence Analysis (MCA) analyses were carried out using Past 3.2 software. The results obtained show that pangolins are mainly exploited for traditional food and medicine. However, the trafficking of their scales has increased demand in the area. The demand for the scales comes mainly from the big cities (Bukavu, Goma, Kisangani) and from some foreign countries (Uganda, Burundi, Tanzania and agents of the United Nations Stabilization Mission in Congo). The trade in scales to satisfy external demand, although still in its infancy, is threatening pangolins in this area where hunting is strictly prohibited. This trade goes through intermediaries, the main ones being local children.
\end{abstract}

(C) 2020 International Formulae Group. All rights reserved.

Keywords: Preliminary study, exploitation, pangolins, Kahuzi-biega, DRC.

\section{INTRODUCTION}

La surexploitation de la faune est l'une des principales pressions à l'origine du déclin des espèces et des extinctions locales (Ingram et al., 2018).

De nos jours, l'augmentation de la demande et du prix de certains produits dérivés des espèces menacées constitue un des grands problèmes de la conservation (Challender et al., 2014).

Le constat est alarmant en ce qui concerne les ressources animales comme les mammifères. Leur exploitation touche même les espèces protégées telles que l'okapi, l'éléphant, les pangolins qui sont régulièrement vendues sous forme de viande boucanée sur le marché de Kisangani, de Yangambi et de Kinshasa (Dudu, 2002; Kambale, 2015 ; Mpamu , 2010).

L'exploitation commerciale est le facteur clé du déclin rapide des populations d'espèces des pangolins d'Asie au cours des dernières décennies (Mambeya et al., 2018). Sans surprise, cela a entraîné une augmentation du commerce international et un trafic de pangolins africains en Asie, principalement pour leurs écailles (Challender \& Hywood, 2012; Challender \& Waterman, 2017).

Les estimations générales stipulent qu'entre $500 \quad 000$ et 2,7 millions des pangolins forestiers sont capturés chaque année pour des fins commerciales (Anonyme I, 2017). Entre 2010 et 2015, 67 pays ont participé au trafic international des pangolins, et chaque année, 27 nouvelles routes pour ce commerce ont été identifiées. Ceci souligne la nature dynamique dudit commerce (Heinrich et al., 2017).

Pour certains auteurs, notamment Garric (2016), Challender \& Waterman (2016) et Arnoud (2017), ces animaux font l'objet d'un braconnage particulièrement intense à destination du marché Sud-Est asiatique. Le fait qu'ils soient plus faciles à attraper, ils seraient parmi les mammifères les plus menacés au monde par le braconnage et le commerce illégal. Leur taux de mortalité est passé de $0,04 \%$ en 1972 à 1,83\% en 2004 (Arnoud, 2017). 
Considérées comme menacées d'extinctions, les quatre espèces des pangolins d'Afrique ont été classées en annexe 1 de la liste rouge de l'IUCN (UICN, 2019).

Pour la République Démocratique du Congo (RDC), Challender \& Waterman (2017) signalent que les données sur les pangolins sont insuffisantes car n'ayant jamais fait d'objet d'étude. Pourtant, l'intérêt de leur protection a atteint les dimensions planétaires. On sait que ces animaux sont exploités depuis toujours comme gibiers par les populations se trouvant dans les zones de leur répartition, bien que les statistiques sur l'exploitation soient inexistantes au niveau des autorités coutumières et de différents services ayant la gestion de la faune dans leurs attributions.

Certes, il faut reconnaître que les pangolins mènent un mode de vie cryptique, ce qui rend leur suivi difficile dans la nature. Aucune étude spécifique n'a porté sur l'exploitation des pangolins dans le Parc National de Kahuzi-Biega (PNKB) en RDC. Or, les saisies des pangolins ont été signalés dans les pays envoisinant la RDC, à savoir le Centrafrique, le Sud-Soudan, l'Ouganda, la Tanzanie et la République du Congo (Heirnrich et al., 2017). Il y a donc lieu de se demander d'où proviennent ces pangolins ou leurs produits secondaires, et qui sont les acteurs impliqués dans ce trafic ?

L'objectif poursuivi dans cette étude est de dégager les différentes raisons pour lesquelles ces animaux sont exploités et identifier les différents acteurs intervenant dans ce domaine via l'information et les connaissances des riverains autour du PNKB.

\section{MATERIEL ET METHODES Milieu d'étude}

La présente étude a été menée dans treize villages se trouvant autour de Tshivanga et Itebero (PNKB). Le PNKB est l'une des aires protégées de la RDC située dans le rift albertin. Il a une superficie de 600.000 ha et est composé de deux parties différentes : d'une part, la haute altitude renfermant la forêt ombrophile. C'est un centre d'endémisme afro-montagnard dont le point culminant est situé sur le mont Kahuzi (3,308 m). De l'autre part, la basse altitude contenant la forêt ombrophile guinéocongolaise dont l'altitude varie entre $700 \mathrm{~m}$ et $1700 \mathrm{~m}$. Il est situé en cheval de trois provinces, notamment le Sud et Nord-Kivu, et le Maniema. Les deux Kivu figurent parmi les provinces les plus habitées en RDC (Hall et al., 1998; Mutimanwa, 2001; ICCN, 2009; UICN, 2010). Il fait partie du Landscape 10 (Maiko-Tayna-Kahuzi-Biega), selon la classification des régions prioritaires de conservation en Afrique centrale (PFBC, 2007). Le parc est administrativement subdivisé en 4 Secteurs à savoir: Lulingu, Njovu, Tshivanga et Itébero.

La présente étude a été menée autour de Tshivanga et Itebero (Figure 1). Le PNKB est l'un des sites le plus important de la région du rift albertin aussi bien pour les espèces endémiques qu'en termes de richesse spécifique. Il compte 136 espèces de mammifères, parmi lesquelles le Gorille de Grauer (G. gorille graueri), le Chimpanzé (Pan troglodytes schweinfurtii), la genette géante (Genetta victoriae), la genette aquatique (Osbornictis piscivora), l'Eléphant de forêt (Loxodonta cyclotis), l'Hylochère (Hylochoerus meinertzhageni), le Bongo (Tragelaphus euryceros), etc. La réserve est située dans une importante zone d'endémisme pour les oiseaux (Endemic Bird Area): 349 espèces d'oiseaux ont été identifiées dont 32 endémiques. Le PNKB est aussi situé dans un Centre d'endémisme pour les plantes : 1178 espèces ont été répertoriées dans la zone de haute altitude (Hart et al., 2007 ; Hall et al., 1997, 1998). Il est parmi les sites les plus importants en termes d'endémisme et d'espèces les plus menacées (Plumptre et al., 2008).

\section{Collecte de données}

La technique d'enquête a été utilisée pour la récolte de données. Elle a consisté à interroger un échantillon de la population vivant dans treize villages répartis dans deux 
zones différentes à savoir: Six villages dans la zone 1 (autour du Secteur Tshivanga), notamment Muyange, Buhungule, Bunyakiri, Kambegete, Hombo Sud et Hombo Nord et sept villages dans la zone 2 (autour du Secteur Itebero), entre autre Kimbaseke, Chambogho, Masanganjiya, Mbughaka, Banakenge, Chabakungu et Walikale centre. Il est important de signaler que dans la zone 1, les interviews individuelles ont été réalisées dans quatre villages, à savoir Bunyakiri, Kambegete, Hombo Sud et Hombo Nord. L'exclusion de Muyange et Buhungule se justifie par le fait que, pendant les entrevues en groupe tenus dans ces deux villages, les pangolins y demeurent des animaux inconnus. Ils y sont confondus avec la tortue, localement appelée «nguru». Le choix des villages avait été orienté par les chercheurs des Wildlife Conservation Society (WCS) conduisant des enquêtes autour du PNKB.

Soulignons que les deux zones d'étude avaient été circonscrites lors des enquêtes pour raison d'efficacité et de comparaison d'opinion. La zone 1 communiquent directement avec la ville de Bukavu (Province du Sud-Kivu, à proximité de la République du Rwanda, la République du Burundi et de la Tanzanie) et la zone 2 (Itebero-Walikale centre) débouche directement sur les Villes de Goma (Province du Nord-Kivu, proche du Rwanda et de l'Ouganda) et de Kisangani (Province de la Tshopo), reliée directement à la Ville de Kinshasa par le fleuve et l'aéroport ; mais aussi connectée par la route à l'Ouganda en passant par les villes de Beni (Nord-Kivu) et Bunia (Province de l'Ituri) proche de l'Ouganda

\section{Les interviews individuelles}

Sur base d'un guide d'entretien, un total de 116 personnes (zone $1: 55$ et zone $2: 61)$ ont été interviewées pour recueillir les informations sur l'exploitation des pangolins autour du PNKB (Figure 2). Les personnes interviewées appartiennent à diverse couche de la population tel que présenté dans le Tableau 1.

Il est important d'indiquer que, l'activité chasse, bien que pratiquée clandestinement, demeure interdite dans les parages du PNKB. Pour ce faire, l'action des éco-gardes est intense dans cette zone. Ceci fait que les riverains pratiquants la chasse ont peur de s'identifier en tant que chasseurs.

Les personnes interviewées étaient choisies à l'aide d'un échantillonnage systématique, en sillonnant les parcelles des villages et en considérant la première parcelle comme celle située à l'entrée d'un quartier avec un intervalle d'une parcelle (soit dans l'ordre 1, 3, 5, 7, etc.). Un effort a été fourni pour interroger les jeunes, les adultes et les vieillards. L'interview se faisait en langue swahili, bien que le questionnaire ait été préparé en français. Les causeries en aparté étaient organisées informellement avec certaines personnes ciblées pour vérifier l'exactitude des informations fournies par les personnes interviewées. Vu le contexte de la zone d'étude (parc), les questions prévues avaient suscité une sensibilité extrême et complexe au sein des certains paysans, si bien que d'autres ont carrément refusé d'être interviewés. Au regard de la complexité des réponses à fournir, l'entretien prenait en moyenne 35 minutes par personnes interrogées.

\section{Interviews en groupe}

Les interviews en groupe ont été organisées dans cinq villages, à savoir: Muyange, Buhungulu, Kambegete, Chambogho et Mbughaka. Les groupes étaient constitués d'un nombre allant de 4 à 8 (Figure 3 a et b). Les questions étaient ouvertes mais non prétablies, dont les principales posées consistaient à savoir si les pangolins existent dans la zone et si oui, comment ils sont considérés dans les différentes cultures locales. Tout dialogue était enregistré dans le carnet de terrain et tout point qui prêtait en confusion était clarifiée séance tenante. 


\section{Méthodes analytiques}

Afin de comparer les résultats, une

$$
\mathrm{Fo}=\frac{n i}{N} \times 100
$$

série d'analyse a été effectuée en recourant aux méthodes univariées pour comparer les opinions des répondants. Il s'agit principalement des Tests Chi-carré $\left(\chi^{2}\right)$ et Fisher exact. En outre, la fréquence d'opinion

(Fo) a été obtenue en utilisant la formule.

dont ni : Nombre d'opinions exprimées par personne interviewée

$\mathrm{N}$ : Nombre total des personnes interviewées.

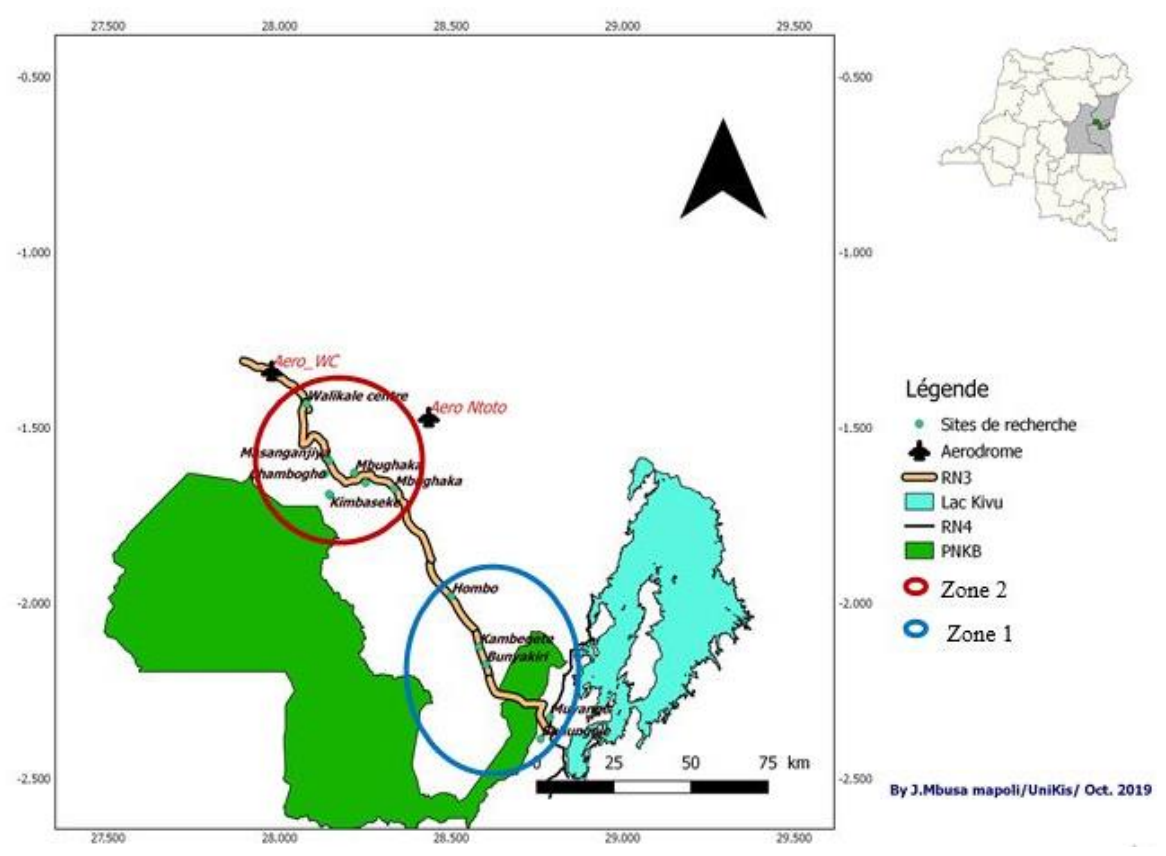

Figure 1 : Localisation des sites d'enquêtes.

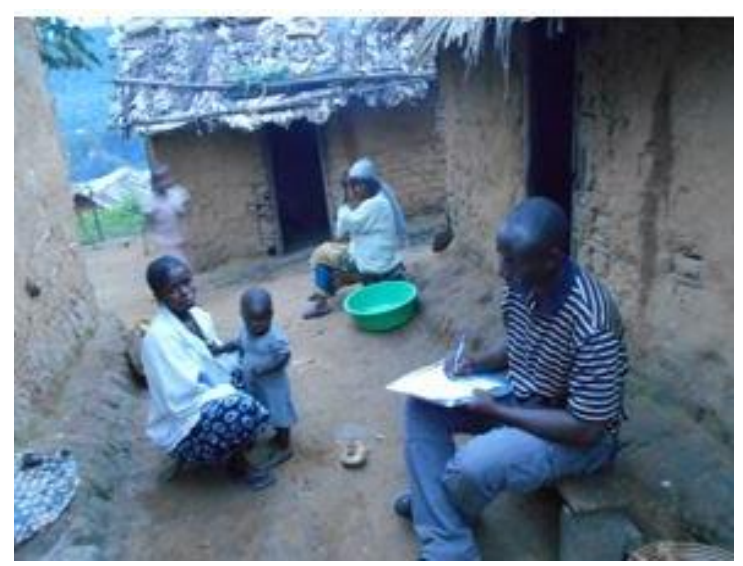

Figure 2 : Interview individuelle à Hombo Nord. 
Tableau 1 : Informations relatives aux personnes interviewées.

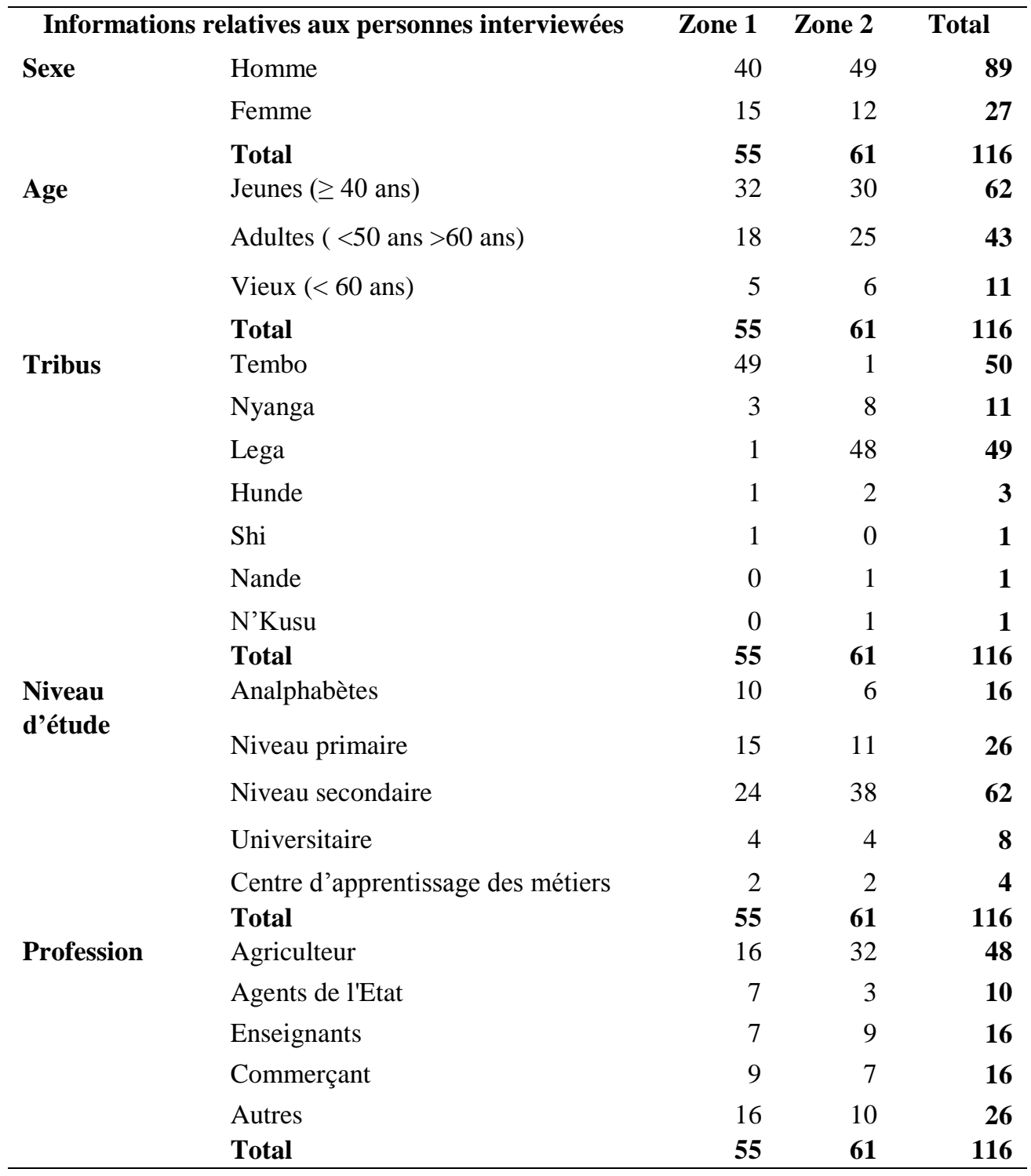
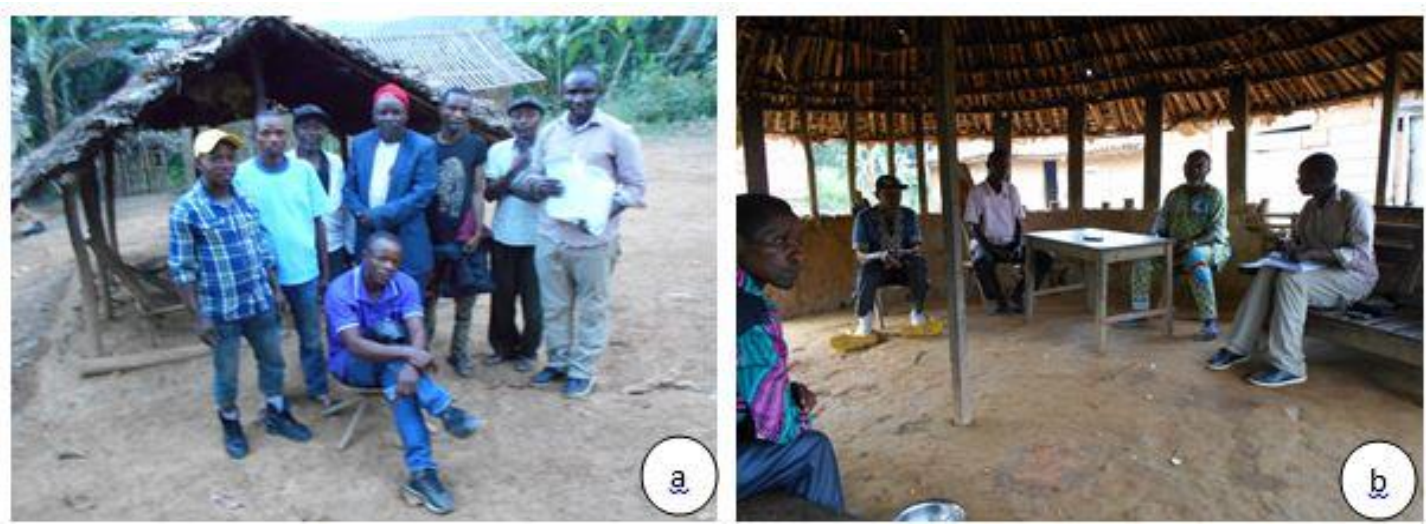

Figure 3 : Focus groups animés à Chambogho (a) et à Mbughaka (b). 


\section{RESULTATS}

\section{Motifs pour lesquels les pangolins sont exploité autour du PNKB}

Autour du PNKB, les pangolins sont exploités pour diverses raisons, principalement l'alimentation, la médecine traditionnelle locale et le commerce des écailles.

D'après la Figure 4, l'alimentation est le stimulus principal pour l'exploitation des pangolins dans la zone 2 (63,9\%). Par contre, dans la zone 1, la médecine traditionnelle est le facteur de base reconnu pour cette exploitation selon $65,4 \%$ des informations reçues. Le commerce n'intervient que pour $2,4 \%$ des cas. Les tests Chi-carré $\left(\chi^{2}=36,2\right.$; $\left.\mathrm{df}=3 ; \mathrm{p}=6,7856 \times 10^{8}\right)$ et Fisher $(\mathrm{p}=$ $\left.9,5129 \times 10^{9}\right)$ montre qu'il existe une différence très hautement significative quant aux raisons qui motivent l'exploitation des pangolins dans les deux zones.

S'agissant de l'alimentation, la majorité des personnes interrogées dans les deux zones $(63,63 \%$ pour la zone 1 et $83,6 \%$ pour la zone 2) mangent ou ont déjà mangé la viande des pangolins. Le résultat du test Chi carré $\left(\chi^{2}=6,0158 ; \mathrm{df}=1 ; \mathrm{p}=0,014178\right)$ a montré que les effectifs de ceux qui mangent la viande des pangolins diffèrent significativement de ceux qui ne la mangent dans les deux zones.

En outre, la majorité des sujets qui mangent la viande des pangolins (zone 1: $34,3 \%$ et zone $2: 43,1 \%$ ) stipulent qu'elle est délicieuse. Par contre, 43,1\% des répondants dans la zone 2 avouent qu'ils mangent cette viande occasionnellement et $22,9 \%$ des sujets interrogés dans la zone 1 ont avoué avoir l'habitude d'en manger. Les test $\mathrm{Chi}^{2}\left(\chi^{2}=\right.$ $12,57, \mathrm{df}=4, \mathrm{p}=0,01)$ et Fisher $(\mathrm{p}=0,008)$ montre que les raisons qui poussent à manger la viande des pangolins diffèrent significativement dans les deux zones.

De ceux qui ne mangent pas la viande des pangolins, $45 \%$ des répondants dans la zone 1 ont dit ne l'avoir jamais trouvé. Par contre, $60 \%$ des répondants dans la zone 2 ont dit être coutumièrement empêchés. Il s'agit essentiellement des membres de la tribu Lega. En effet, pour cette tribu, le pangolin géant est un animal sacré, et pour les deux autres pangolins, il y a beaucoup de conditions pour en manger la viande.

Le résultat du test Chi deux $\left(\chi^{2}=\right.$ $13,12 ; \mathrm{df}=4 ; \mathrm{p}=0,01)$ montre que les raisons évoquées pour ne pas manger la viande des pangolins diffèrent significativement dans les deux zones. Si le pangolin géant est moins consommé, il n'en est pas le cas pour les deux autres espèces.

\section{Usage en médecine traditionnelle locale}

La majorité des répondant $(80 \%)$ dans zone 1 reconnaissent des vertus médicinales aux produits secondaires issus du pangolin (écailles, griffes et os de la queue). Par contre, $66 \%$ des sujets interrogés dans la zone 2 n'en réconnaissent pas.

Les différentes maladies traitées et le mode d'usage sont présentés dans le Tableau 2.

Du Tableau 2, il ressort que les écailles sont les plus utilisées dans la médecine traditionnelles locales, car sur sept maladies identifiées, elles interviennent dans le traitement de quatre. En outre, pour le traitement, chaque maladie a un mode de préparation bien approprié.

\section{Commercialisation des produits secondaires des pangolins}

Il convient d'indiquer que $45 \%$ des répondant dans la zone 1 confirment qu'il y a une demande externe. Par contre, dans la zone 2, 59,0\% des sujets interviewés ne sont pas informés sur cette demande. En plus, dans les deux zones, la majorité des répondants $(56,4 \%$ et $54,1 \%$ ) n'ont jamais entendu parlé du commerce des écailles. Les tests Chi-deux $\left(\chi^{2}\right.$ $\left.=19,55 ; \mathrm{df}=2 ; \mathrm{p}=5,6762 \times 10^{-5}\right)$ et de Fisher $\left(\mathrm{p}=1,3676 \times 10^{-5}\right)$ indique que les personnes interrogées dans les deux zones sont très différemment informées sur l'existence d'une demande externe des pangolins. 
La demande externe des pangolins autour du PNKB est motivée par la rechreche des éléments suivants (Figure 5):

De par la Figure 5, il est ressorti que bon nombre de sujets interrogés dans la zone 1 , soit $40 \%$ indiquent que les demandeurs externes cherchent les écailles des pangolins. Par contre, $48 \%$ de personnes interviewées dans la zone 2 ont stipulé que les demandeurs cherchent les pangolins vivants et $28 \%$ ont évoqué les écailles. Le résultat du test Chicarré $\left(\chi^{2}=7,83 ; \mathrm{df}=5 ; \mathrm{p}=0,16\right)$ et le test de Fisher $(\mathrm{p}=0,15)$ indique que les motifs de demande externe sont quasi les mêmes dans les deux zones.

\section{Qualités des acheteurs des écailles}

Ceux qui ont déjà entendu parler du commerce des écailles ont donné la qualité des demandeurs (Figure 6).

La Figure 6 renseigne que $79,1 \%$ des répondants dans la zone 1 soutiennent que les acheteurs sont des tradipraticiens. Par contre, dans la zone 2 , ce sont plus les trafiquants qui cherchent les pangolins et/ou leurs produits secondaires, ce que $39,3 \%$ des répondants ont avoué. Il est important de signaler aussi que dans la zone 2, environ 17,9\% des répondants ont pointé les agents de la Mission de Nations-Unies pour la Stabilisation de République Démocratique du Congo (MONUSCO) parmi les demandeurs des pangolins et/ou de leurs produits secondaires. Le test Khi-deux $\left(\chi^{2}=17,18 \mathrm{df}=6, \mathrm{p}=0,008\right)$ et le test Fisher $(p=0,001)$ montre que les acheteurs connus dans la zone 2 diffèrent très significativement de ceux connus dans la zone 1.

\section{Facteurs favorisant l'exploitation des pangolins autour du PNKB}

Les principaux facteurs favorisant l'exploitation des panagolins autour du PNKB sont présentés dans la Figure 7.

L'analyse à composante multiple montre que les principaux facteurs favorisant l'exploitation des pangolins autour du PNKB sont l'origine des acheteurs $(\mathrm{OA})$ et le moyen de transport (MT). A cela s'ajoute le problème d'application de la loi (EA) faisant penser à beaucoup d'individus que l'exploitation ne pose pas problème dans la zone, la demande externe (DE) et le commerce des écailles (VE).

\section{Provenance des acheteurs des produits pangolins}

Dans les parages de Tshivanga et Itebero, les acheteurs des produits pangolins sont de deux origines. Il y a les nationaux (congolais) résidants principalement dans les grands centres urbains se trouvant dans le rayon du PNKB, et les étrangers, des nationalité diverses.

\section{Provenance des acheteurs nationaux}

La repartition des acheteurs congolais selon leurs provenances est présentée dans la Figure 8.

Il ressort de la Figure 8 que les acheteurs congolais (RDC) des produits pangolins viennent généralement de Bukavu (66,6\% des réponses en zone 1 ) et de Goma (23,8\% des réponses zone 1). Par contre, $33,3 \%$ des répondants dans la zone 2 ont avoué que les acheteurs viennent de Kisangani et Goma ( $28,6 \%$ de reponses). Le test Chideux $(\chi 2=18,6 ; d f=6 ; p=0,004)$ et le test Fisher $(\mathrm{p}=0,0005)$ montrent que la différence des origines des acheteurs congolais est très significative dans les deux zones.

\section{Nationalités des acheteurs étrangers}

Les acheteurs étrangers viennent de plusieurs pays tel qu'indiqué sur la Figure 9.

La Figure 9 révèle que $100 \%$ des répondants de la zone 1 ayant accepté l'implication des étrangers dans le commerce des écailles, citent les Ougandais et les Burundais. Par contre, 50\% d'entre ceux de la zone 2 citent les agents de la MONUSCO, sans toutefois préciser leurs pays d'origine. En effet, la plupart des casques bleus actuellement et antérieurement basés en Territoire de Walikale sont d'origine asiatique (népalais, indiens, pakistanais, etc.) Les tests 
Chi-deux $\left(\chi^{2}=2,7 ; \mathrm{df}=3 ; \mathrm{p}=0,4\right)$ et Fisher $(p=0,5)$ montrent qu'il n'y a pas de différence statistiquement significative de l'intervention des étrangers dans le commerce des produits pangolins dans les deux zones.

\section{Moyen de transport}

La Figure 10 présente comment le transport est assuré localement pour appuyer le trafic des produits pangolins.

Partant de la Figure 10, la moto est le moyen de transport le plus utilisé dans les deux zones : zone $1: 47,2 \%$ et zone $2: 62,3 \%$ des réponses reçues. L'avion intervient aussi dans le transport selon 3,3\% des répondants dans la zone 2. Les tests Chi-deux $\left(\chi^{2}=17,6\right.$; $\mathrm{df}=4 ; \mathrm{p}=0,001)$ et Fisher $(\mathrm{p}=0,0001)$ montre que le transport est différemment assuré dans ces deux zones.

\section{Organisation du marché des produits pangolins}

Les personnes interviewées ayant déjà entendu parler du commerce des écailles dans les deux zones ont donné les informations ci- après : $41,7 \%$ des répondants dans la zone 1 indiquent que le marché se passe du chasseur au client. Par contre, dans la zone 2, 67,9\% d'eux affirment que le marché se passe du chasseur au client en passant par les intermédiaires. Les tests Chi-deux $\left(\chi^{2}=17,15\right.$; $\mathrm{df}=4 ; \mathrm{p}=0,001)$ et Fisher $(\mathrm{p}=0,0002)$ montrent que le marché d'écailles des pangolins est différemment organisé dans les deux zones.

Ces informations ont permis d'établir un diagramme simplifié et hiérarchisé de l'organisation du marché, telle que la Figure 11 l'illustre.

La Figure 11 montre la hiérarchisation organisationnelle du marché des écailles des pangolins. Le chasseur va en forêt pour tuer les pangolins (1). Il revient avec la viande et les écailles au village où il trouve les acheteurs locaux ou les autres intermédiaires (2), lesquels acheminent les produits achetés dans les centres urbains les plus proches (3) où on trouve soit, les tradipraticiens locaux (preneurs d'écailles) (4), soit les trafiquants pour alimenter le marché externe (4').

\section{由Zone 1 Z Zone 2}

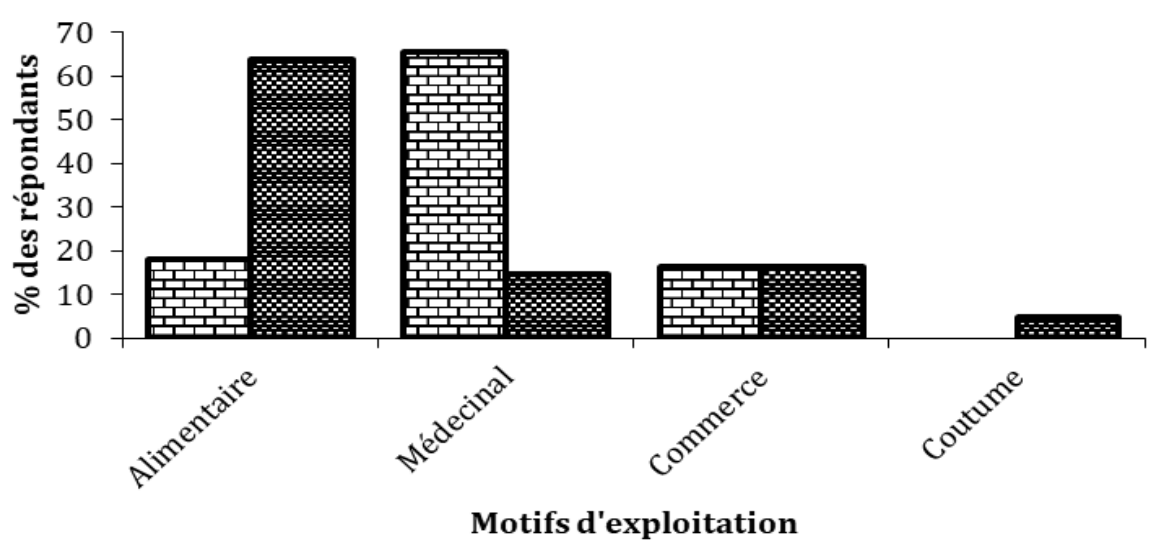

Figure 4 : Principales motivations pour l'exploitation des pangolins. 
Tableau 2 : Maladies traitées par les produits secondaires des pangolins.

\begin{tabular}{|c|c|c|c|}
\hline $\mathbf{N}^{\circ}$ & $\begin{array}{c}\text { Produits } \\
\text { secondaires }\end{array}$ & Maladie traitée & Mode d'usage/préparation \\
\hline \multirow[t]{4}{*}{1.} & Ecailles & Menace d'avortement & $\begin{array}{l}\text { Disposer les écailles en collier sur un fil du palmier, } \\
\text { ensuite enroulé au niveau de la hanche de la femme } \\
\text { grosse menacée d'avortement. }\end{array}$ \\
\hline & & Fontannelle & $\begin{array}{l}\text { Incinérer, puis moudre. Ensuite, mélanger la cendre } \\
\text { avec l'huile de ricin ou de palme, puis appliquer sur } \\
\text { la fontanelle. }\end{array}$ \\
\hline & & Paludisme & $\begin{array}{l}\text { Bouillir les écailles avec d'autres feuilles sauvages } \\
\text { (non précisées) puis enfermer le patient dans un } \\
\text { endroit hermetiquement fermé pour qu'il aspire la } \\
\text { chaleur issue de la préparation. }\end{array}$ \\
\hline & & Maladies demoniaques & Talisman. \\
\hline \multirow[t]{2}{*}{2.} & Griffes & Toux & $\begin{array}{l}\text { Bouillir avec les feuilles des certaines plantes } \\
\text { sauvage (non précisées), puis boire chaud } \\
\text { (Décoction). }\end{array}$ \\
\hline & & Coliques & Décoction. \\
\hline 3. & $\begin{array}{l}\text { Os de la } \\
\text { queue }\end{array}$ & $\begin{array}{l}\text { Lombalgie et douleurs } \\
\text { de la colonne } \\
\text { vertébrale et du dos }\end{array}$ & $\begin{array}{l}\text { Incinerer l'os de la queue, puis mélanger la cendre } \\
\text { avec de l'huile de ricin ou de palme et appliquer } \\
\text { comme une pomade. }\end{array}$ \\
\hline
\end{tabular}

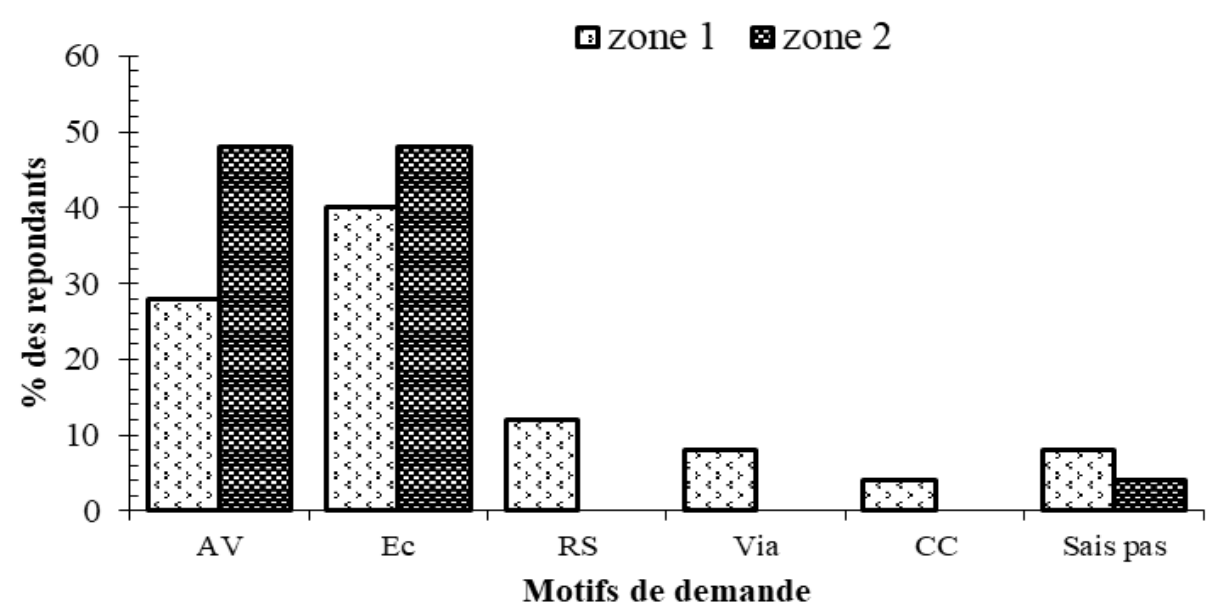

Figure 5 : Motifs de demande externe des pangolins et/ou produits secondaires. $\mathrm{AV}=$ animal vivant, $\mathrm{Ec}=$ Ecailles seulement, $\mathrm{RS}=$ Recherche scientifique, $\mathrm{CC}=\mathrm{Cé}$ rémonies coutumières. 


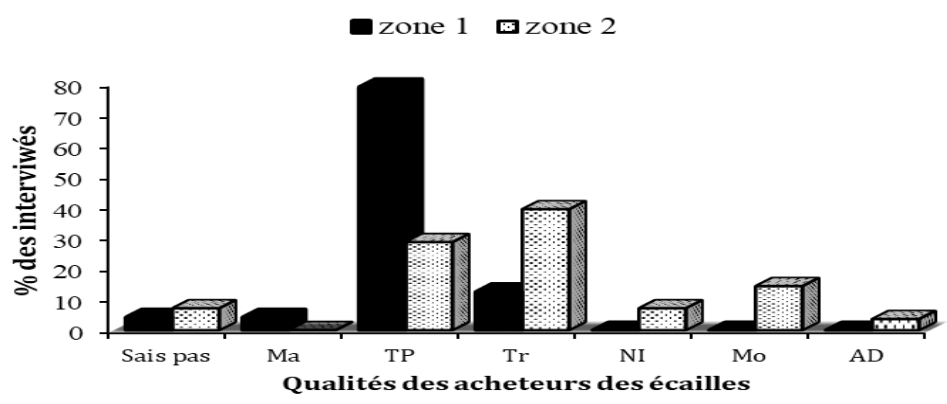

Figure 6 : Qualité des acheteurs d'écailles.

$\mathrm{Ma}=$ Malade, $\mathrm{TP}=$ Tradipraticiens, $\mathrm{Tr}=$ Trafiquants, $\mathrm{NI}=$ Non identifié, $\mathrm{Mo}=$ Agent $\mathrm{MONUSCO}, \mathrm{AD}=$ Artiste décorateur

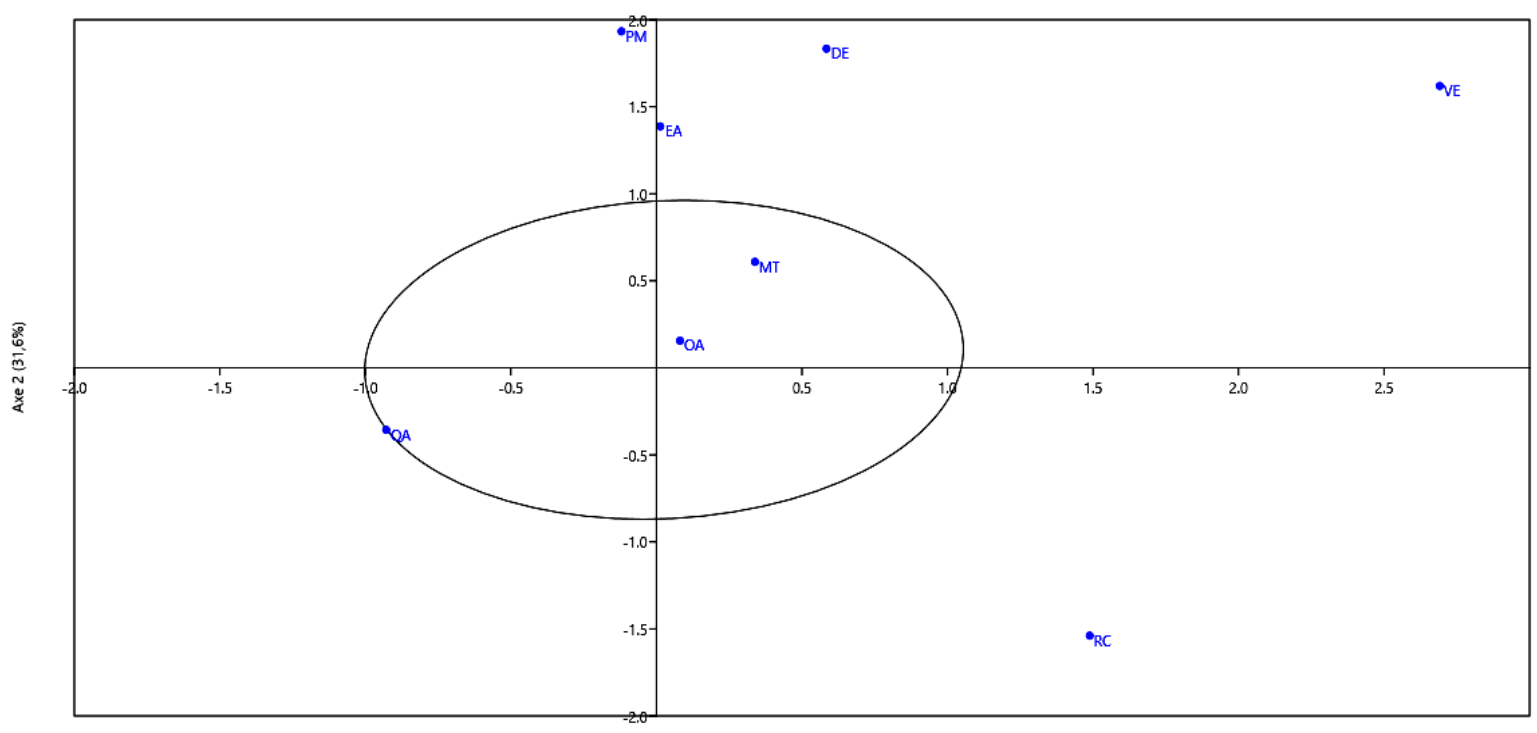

Axe $1(38,5 \%)$

Figure 7: Dispersion des facteurs influançant l'exploitation des pangolins autour du PNKB. $\mathrm{OA}=$ Origine des acheteurs, MT= Moyen de transport, $\mathrm{QA}=$ Qualité des acheteurs, $\mathrm{RC}=$ Raison de consomation, $\mathrm{EA}=$ l'exploitation est autorisée dans la zone, $\mathrm{PM}=$ Propriétés médicinales, $\mathrm{DE}=$ demande externe, $\mathrm{VE}=$ Ventre des écailles.

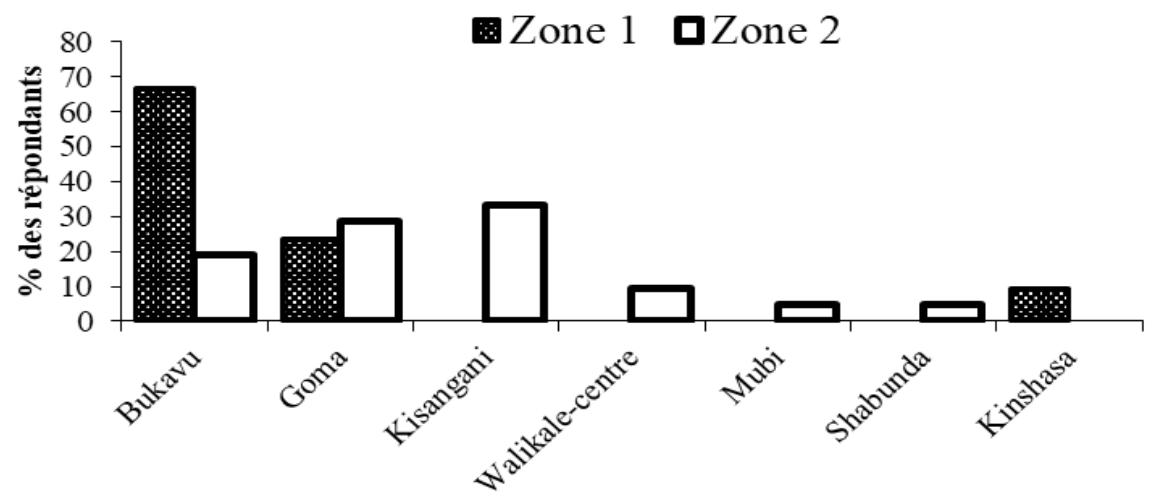

Ville d'origine/ Zone

Figure 8: Villes d'origine des acheteurs nationaux. 


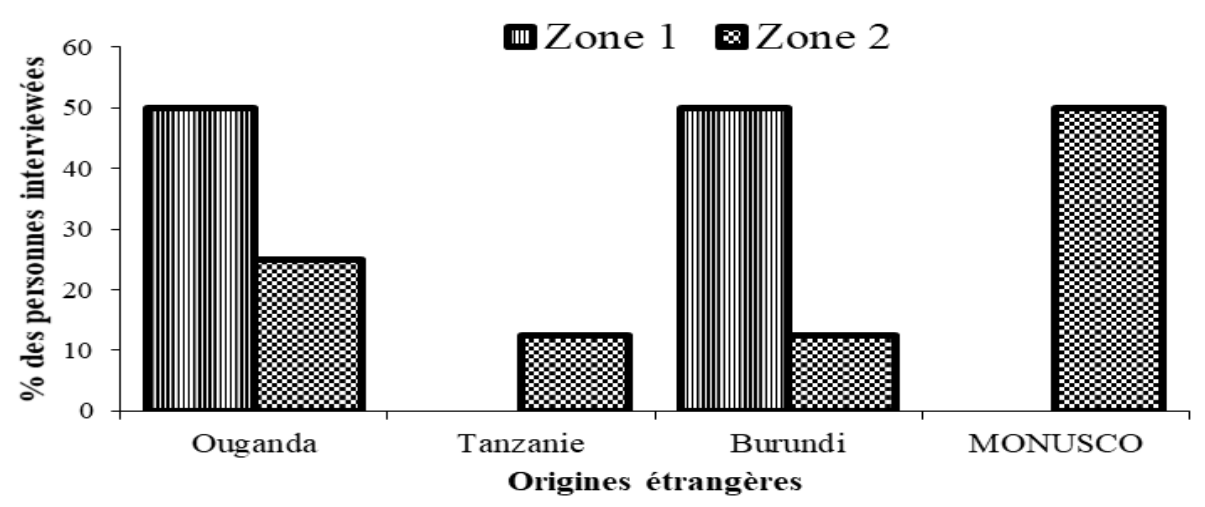

Figure 9: Pays d'origine des acheteurs étrangers.

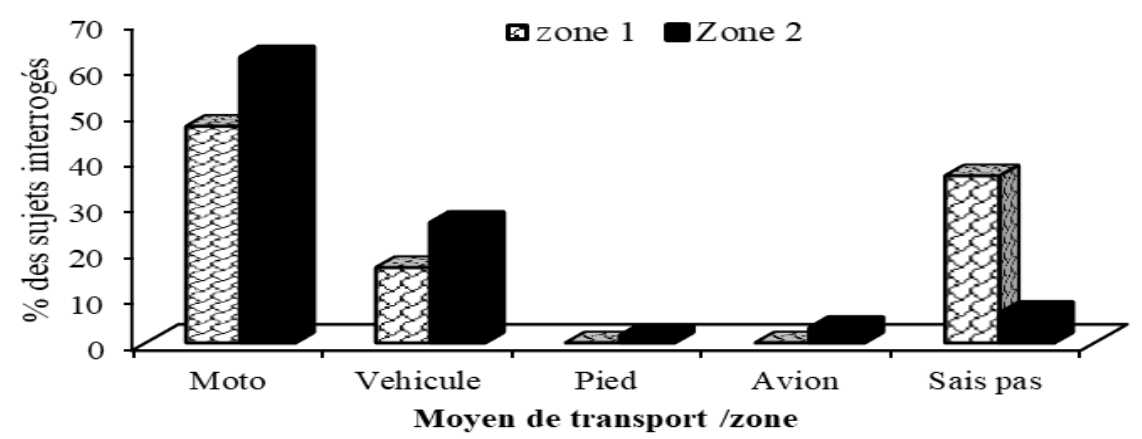

Figure 10: Moyens de transport courant dans la zone d'étude.

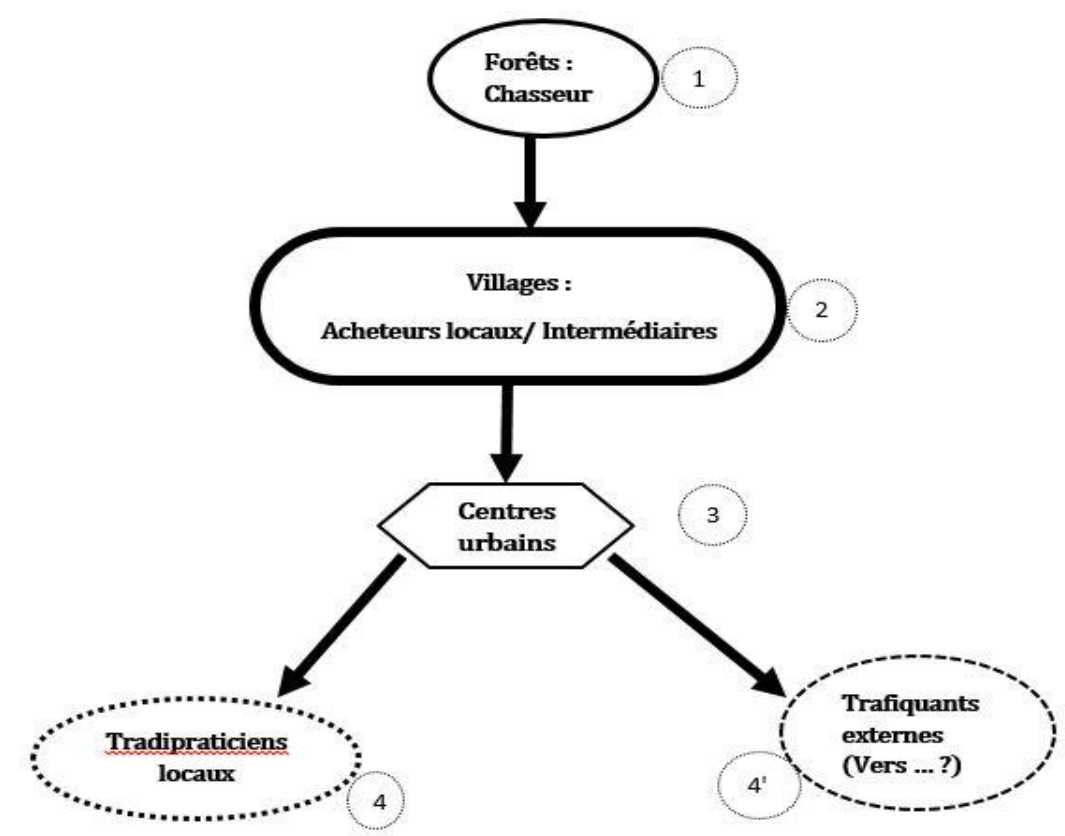

Figure 11: Organisation hierarchique du marché des écailles des pangolins. 


\section{DISCUSSION \\ Information relatives aux personnes interviewées}

En rapport avec les personnes interviewées, l'âge est parmi les variables qui ont attirées beaucoup l'attention. Les intervalles prédominants sont compris entre 20 et 30 ans pour la zone 1 et de 40 à 50 ans pour la zone 2. Ceci témoigne que la population est encore jeune. C'est à la fois un danger et un atout. Le fait que la population soit encore jeune, cela constitue un danger car les jeunes, étant actifs, sont en perpétuel quête des ouvertures pour gagner de l'argent. De ce fait, ils cherchent toutes les informations possibles sur toute chose susceptible d'être exploitée pour des raisons lucratives. Ils sont aujourd'hui informés de la valeur que représentent le pangolin et ses écailles dans le commerce international. C'est pourquoi certains d'entre ces jeunes commencent à se livrer à leurs chasses. Une population jeune est un atout, car elle peut être utilisée dans la sensibilisation pour lutter contre l'expansion de la chasse aux pangolins dans ce milieu, avant qu'elle n'y atteigne des dimensions incontrôlables.

\section{Motifs pour l'exploitation des pangolins}

Les motifs pour lesquels ces animaux sont exploités diffèrent cependant d'une zone à l'autre $\left(\mathrm{p}=1,7243 \cdot 10^{-16}\right)$.

Dans la zone 1, la majorité des sujets interviewés ont indiqué qu'ils sont principalement exploités pour la médecine traditionnelle locale, alors que dans la zone 2 , c'est plutôt pour l'alimentation qui est le stimulus principal.

Les produits secondaires des pangolins (écailles, griffes et os de la queue) sont utilisés dans le traitement de beaucoup de maladies, entre autre : menace d'avortement, paludisme, maux du dos, coliques, fontanelle, voire chasser les mauvais sorts et procurer la protection.

S'agissant de l'alimentation, la majorité des répondant dans les deux zones, mangent ou ont déjà mangé la viande des pangolins . Diverses raisons justifient le fait de manger la viande des pangolins, entre autres, le gout délicieux, l'habitude, viande occasionnelle et la curiosité. Le test $\mathrm{Chi}^{2}$ $(\mathrm{p}=0,005)$ a montré qu'il y a une différence significative.

$\mathrm{Au}$ demeurant, de ceux qui ne mangent pas cette viande, $45 \%$ dans la zone 1 ne l'ont jamais trouvée et $35 \%$ ont dit être empêchés par leur foi (réligion). Par contre, $60 \%$ des répondants dan la zone 2 sont coutumièrement empêchés. Il s'agit essentiellement des Lega.

En effet, pour cette tribu, le pangolin géant est un animal sacré, et pour les autres pangolins, il y a beaucoup de conditions pour le manger. Le test de Kruskal-Wallis $(\mathrm{p}=0,44)$ montre qu'il n' y a pas de différence significative entre les deux axes. Selon les auteurs Boakye et al. (2016), Waterman et al. (2014a, b, c), Pietersen et al. (2014), Gaubert (2011) et Soewu \& Ayodele (2009), les pangolins d'Afrique sont tous menacés par la chasse pour les marchés locaux. Ils sont soumis à une exploitation importante et souvent intensive pour la viande de brousse consommée localement et la médecine traditionnelle. Pour Ichu et al. (2017), La quête des pangolins pour nourrir la demande intérieure en viande et la demande internationale en écailles motive fortement les chasseurs, qui exercent alors une pression immense sur ces espèces déjà très vulnérable.

La viande des pangolins est une source de protéines pour de nombreux ménages locaux car elle est réputée pour son goût. C'est également une source de revenus car le pangolin est parfois entièrement vendu avec des écailles ou découpé en tranches plus petites et plus abordables, préparées et vendues sur les marchés du village.

Usage des produits secondaires des pangolins dans la médecine traditionnelle locale

Dans la zone 1, la majorité des répondant reconnaissent des vertus médicinales aux produits provenant des pangolins. Par contre, dans la zone 2, la majorité a refuté cette affirmation.

Les griffes, l'os de la queue et surtout les écailles sont les plus utilisées dans la médecines traditionnelles locales. Ces produits traitent diverses maladies, entre autres, les ménaces d'avortement, la fontannelle, le palludisme, la toux, les 
coliques, les douleurs du dos et de la colonne vertébrale, etc.

En outre, il y'a un mode de préparation bien approprié pour chaque produit et chaque maladie. Ces résultats concordent avec ceux de Boakye et al. (2015) et Zhao et al. (2015) qui ont stipulés que les écailles des pangolins guérissent plusieurs maladies dont le cancer grave, rhumatisme, convulsion, gastrite. Au Siéra à Leone, elles traitent 59 maladies. En Chine, elles sont utilisées dans la détoxication et comme stimulant du lait maternel. De surcroît, en Tanzanie le pangolin est appelé «Bwana muganga» car chaque partie du corps soigne au moins une maladie (Gaubert, 2011). D'ailleurs, dans la vallée du Great Ruaha (Tanzanie), Walsh (2007) indique qu'il fournit des ingrédients pour diverses sortes de préparations médicinales et magiques. Ses écailles sont de loin les plus utilisées à des fins curatives (par exemple dans le traitement du panaris, du torticolis, des douleurs au dos, de la pneumonie, des convulsions d'enfants, des érythèmes) et pour la fabrication d'amulettes (en particulier pour protéger les chasseurs et leurs camps des bêtes sauvages, des gardes-chasse et d'autres agents de malheur).

\section{Demande externe, origines et qualité des acheteurs, et moyen de transport}

La demande externe dans les deux zones a été confirmée la plupart des personnes interrogées. Les acheteurs sont de deux origines : les nationaux et les étrangers. Même si le test Khi-carré $(\mathrm{p}=0,001)$ a indiqué que les enquêtés dans les deux axes sont différemment informés par rapport à la demande externe des pangolins, pendant les focus group, les participants ont confirmé qu'une demande externe des produits pangolins existe déjà dans la zone. S'agissant de l'origine des acheteurs externes, la plupart des répondants ayant reconnu cette demande, soit $40 \%$ et $29,5 \%$, ont indiqué que ce sont des nationaux. Ils viennent généralement de Bukavu, Kisangani, Goma et Walikale centre. La différence est très significative dans les deux axes $(\mathrm{p}=0,009)$. Seul $3,27 \%$ des répondants dans la zone 2 ont évoqué les étrangers. Tous les répondants dans la zone 1 ayant reconnu l'implication des étrangers dans la demande des écailles, indexent les Ougandais et les Burundais. Par contre, dans la zone 2, la majorité des répondants, soit $62,5 \%$ d'enquêtés, a cité les agents de la MONUSCO, sans présiser leurs pays d'origines. Ceci se justifierait par le fait que les casques bleus se trouvant ou ayant sejourné en Territoire de Walikale sont majoritairement d'origine asiatique (Nepalais, Indiens, Pakistanais, Bangladais, Chinois, etc.). $12,5 \%$ ont ajouté les tanzaniens. Le test Khi-deux $\left(\chi^{2}=10, \mathrm{df}=3, \mathrm{p}=0,01\right)$ montre que l'intervention des étrangers dans le commerce des écailles est différente dans les deux axes.

L'implication des ougandais, des burundais et Tanzaniens se justifierait par le fait qu'ils partagent leurs frontières avec l'Est RDC et sont facilement accessible à partir de Goma (Ouganda) ou de Bukavu (Burundi et Tanzanie).

A propos des qualités des acheteurs, dans la zone 1, c'est majoritairement des tradipraticiens, alors que dans la zone 2, il s'agit plutôt des trafiquants. Parmis les acheteurs, dans la zone 1, les uns cherchent les écailles, par contre dans la zone 2, la demande est plus cumulative. $40 \%$ d'enquêtés stipulent que les demandeurs cherchent les aniamux vivants et les écailles. En effet, selon les auteurs Boykye et al. (2015), Nash et al. (2016), Heinrich et al. (2017) et Karawita et al. (2018), les pangolins sont actuellement parmi les mammifères les plus victimes du commerce illégal des espèces sauvages. Ils sont surexploités pour le commerce international et pour leur utilisation locale, principalement pour leur viande et leurs écailles.

En outre, l'Ouganda, le Burundi et la Tanzanie figurent parmi les pays à la fois producteurs et intermédiaire dans le trafic des écailles des pangolins (Jaramogi, 2017). Faisant ses études sur la viande des brousses, Fargeot (2008) souligne qu'en CentreAfrique, $78 \%$ des pangolins sont vendus vivants sur le marché des viandes de brousses. Gevers (2017) indique que les pangolins sont les plus chassés après l'éléphant et prend une proportion croissante dans la liste des animaux chassés en Afrique Centrale. L'état de la route reliant la zone 1 à la ville de 
Bukavu ne permet que l'usage de la moto comme moyen de transport. Ceci expliquerait le faible trafic des produits issus des pangolins. Par contre, dans la zone 2, les routes reliant Walikale centre aux villes de Kisangani et Goma sont plus ou moins praticables, facilitant ainsi l'arrivé des véhicules de toute sorte.

L'on note aussi l'existence des pistes d'atterrissage à Walikale centre et ses environs. Ces infrastructures faciliteraient le trafic des produits pangolins.

C'est ainsi que Baye-Niwah et al. (2020) avaient indiqué que les pratiques anthropiques et leurs conséquences sur l'environnement sont au centre de l'actualité à ces jours.

\section{Organisation du marché des produits pangolins}

L'hierarchisation du marché va du chasseur au client en passant par des intermédiaires. En fait, le chasseur va en forêt pour tuer les pangolins. Il revient avec la viande et les écailles au village où il trouve les acheteurs locaux ou les autres intermédiaires, lesquels acheminent les produits achetés dans les centres urbains les plus proches. Ici, on trouve soit les tradipraticiens locaux (preneurs des éailles), soit les trafiquants pour alimenter le marché externe.

Le transport est généralement assuré par les motos, véhicules, mais aussi les avions. Généralement dans l'organisation du marché, il y a un collecteur villageois (venant le plus souvent des centres urbains) qui concentre les produits du village et le vend à un revendeur urbain qui approvisionne les preneurs (Robinson \& Redford, 1994; Diéval, 2000) ; ce qui fait qu'à la sortie de la forêt, le produit est transporté par tous les moyens modernes disponibles (Mpamu, 2010). Au Cameroun, une grande partie des écailles est vendue sur le marché noir, tandis que certains praticiens les utilisent également pour la médecine traditionnelle (Ichu et al., 2017). La demande accrue de la Chine a fait des pangolins le mammifère le plus victime de la traite dans le monde, ont martelé Trageser et al. (2017). L'exploitation commerciale est le facteur clé du déclin rapide des populations d'espèces de pangolin d'Asie au cours des dernières décennies (Mambeya et al., 2018). Sans surprise, cela a entraîné une augmentation du commerce international et un trafic de pangolins africains en Asie, principalement pour leurs écailles (Challender \& Hywood, 2012; Challender et Waterman, 2017). Au Gabon, les travailleurs asiatiques de l'industrie achètent des pangolins directement à des chasseurs (Mambeya et al., 2018). Actuellement, on observe une surexploitation de la faune mammalienne à la suite d'une chasse commerciale (Laurance et al., 2006). Malheureusement cette chasse commerciale est accusée de mettre en péril la vie animale dans les forêts d'Afrique centrale et, à terme, de menacer l'ensemble de cet écosystème (Fargeot, 2004).

\section{Conclusion}

$\mathrm{Au}$ terme de cette étude, pionnière dans la zone, il convient d'indiquer que l'exploitation des pangolins et de ses produits secondaires dans l'hinterland du PNKB est au départ une exploitation de substance. Les paysans les exploitent pour l'alimentation et la médecine traditionnelle. Cependant, la demande de leurs produits secondaires pour alimenter les marchés SudEst asiatiques, est à la base d'une menace de plus à plus accrue.

Les enquêtes menées autour du PNKB révèlent, actuellement que les pangolins sont clandestinement exploités pour le commerce de leurs écailles, suite à la demande externe dont les demandeurs viennent des milieux urbains. Ceci représente déjà une menace au niveau local.

Il est aussi important de souligner que la médecine traditionnelle locale utilisant les écailles; et la viande, jugée savoureuse sont parmi les facteurs principaux favorisant cette exploitation. Au regard des informations recueillies auprès de personnes interviewées, il est probable que l'ampleur du trafic des pangolins et de leurs écailles soit élevée.

Malheureusement, aucune preuve de saisie au niveau des services ayant dans leurs attributions le contrôle dans la faune n'a été réalisée. Cela fait penser que la clandestinité favorisant cette exploitation ferait intervenir certains agents impliqués 
dans la surveillance, surtout que la chasse est strictement interdite dans la zone autour du PNKB.

Pour ce faire, des études intenses devraient être menées dans la zone évaluer l'état de lieu et les menaces sur les populations des pangolins dans la zone, mais aussi pour comprendre en fond les acteurs clés impliqués réellement dans cette demande externe. Des intenses sensibilisations devraient aussi être envisagées le plutôt possible dans cette zone, avant que la jeunesse locale ne soit complétement séduite pour coopérer avec les trafiquants.

Il est aussi impérieux que la RDC, en tant que signataire de la convention de Washington (CITES), se mette à appliquer scrupuleusement la loi sur la faune; que les partenaires financiers et techniques conjuguent les efforts pour comprendre les tenants et les aboutissants du trafic des pangolins en RDC et que la surveillance soit renforcée pour arriver à sauver ce qui reste, le plus tôt possible.

\section{CONFLIT D'INTERETS}

Les auteurs déclarent qu'ils n'ont pas de conflit d'intérêts.

\section{CONTRIBUTION DES AUTEURS}

Pour la réalisation de cette étude KMD et KVB ont contribué dans la mise en place du questionnaire d'enquête et ont participé au dépouillement des données. MIJC et GMS ont contribué à l'analyse et au traitement des données. Quant à KNJ et TTF ont fait un plaidoyer auprès de US FISH Wildlife pour financer la présente étude, et ont donné des observations pour l'amélioration du manuscrit.

\section{REFERENCES}

Baye-Niwah C, Todou G, Souare K, Abdoulaye A, Bay S, Atem E. 2020. Diversité et usages des plantes ligneuses des agrosystèmes périphériques de la ville de Maroua (Extrême-Nord, Cameroun). Int. J. Biol. Chem. Sci., 14(3): $\quad 966-982 . \quad$ DOI : https://doi.org/10.4314/ijbcs.v14i3.25
Boakye MK, Kotzé A, Dalton DL, Jansen R. 2016. Unravelling the pangolin bushmeat commodity chain and the extent of trade in Ghana. Hum. Ecol., 44: 257-264.

Boakye MK, Pietersen DW, Kotze A, Dalton DL, Jansen R. 2015. Knowledge and uses of African pangolins as a source of traditional medicine in Ghana. Plos One, DOI:

http://dx.doi.org/10.1371/journal.pone.01 17199.

Challender D, Hywood L. 2012. African pangolins under increased pressue from poaching and intercontinental trade. TRAFFIC Bull., 24: 53-55.

Challender D, Nguyen TV, Shepherd C, Krishnasamy K, Wang A, Lee B, Panjang E, Fletcher L, Heng S, Ming SHJ, Olsson A, Nguyen TTA, Nguyen QV, Chung YF. 2014. Manis Javanica. The IUCN Red List of Threatened Species 2014.2 DOI : https://doi.org/102305/IUCN.UK.20142.RLTS.T12763A45222303.

Challender DW, Waterman C, Baillie JEM. 2014. Scaling up pangolin conservation. IUCN SSC pangolin specialist group conservation action plan. ZSL. London, UK.

Challender DW. 2013. The most traded wild mammal - the pangolin - is being eaten to extinction. IUCN. https://www.iucn.org/content/mosttraded - wild-mammal-pangolin-being-eatenextinction. Accessed on December 10, 2017.

Challender DWS, Baillie JEM, Waterman C. 2012. Catalysing conservation action and raising the profile of pangolins - the IUCN SSC Pangolin Specialist Group (PangolinSG). Asian Journal of Conservation Biology, 1(2): 140-141.

Challender DWS, Waterman C. 2017. L'application des décisions CITES 17.239 b) et 17.240 relatives aux pangolins (Manis spp.) Rapport IUCN.

Cota-Larson R. 2017. Guide d'identification rapide des espèces de pangolin: Outil rapide d'évaluation pour le terrain et le bureau. Préparé pour l'Agence des ÉtatsUnis pour le développement international (United States Agency for 
International Development). Bangkok : USAID Wildlife Asia Activity. Disponible en ligne à : http://www.usaidwildlifeasia.org/resourc es.

Diéval S. 2000. La filière viande de chasse à Bangui, République Centrafricaine. Mémoire de fin d'étude, ISTOM. CergyPontoise, France. 211 p.

Fargeot C. 2004. La chasse commerciale en Afrique centrale I. la venaison ou le négoce d'un produit vivrier. Bois et Forêts des Tropiques, 282 (4) : 27-40.

Fargeot C. 2008. Le commerce de la viande de chasse en Afrique Centrale. Etude d'un marché-porte : le PK 12 à Bangui (RCA), CIRAD, Montpellier, France, PDF, 12p.

Gevers L. 2017. Les pangolins victimes d'une chasse sans merci en Afrique Centrale, https://www.sciencesetavenir.fr/animaux /une-etude-revele-1-impact-de-la-chassesur-les-pangolins-d-afrique-

centrale_114933, consulté le 5 Novembre 2018 à 14 h00.

Hall JS, Inogwabini BI, Williamson EA, Omari I, Sikuabwaboa C, White LJT. 1997. A survey of elephants (Loxodonta africana) in Kahuzi-Biega National Park, Lowland sector in Eastern Zaïre. African Journal of Ecology, 35 : 213-223.

Hall JS, White LJT, Inogwabini BI, Omari I, Morland HS, Williamson EA, Saltonstall K, Walsch P, Sikuabwaboa C, Dumbo B, Kaleme PK, Vedder A, Freeman K. 1998. Survey of Grawer's gorilla (Gorilla gorilla graweri) and Easter Chimpanzes (Pan troglodytes schweinfurthi) in Kahuzi-Biega National Park Lowland Sector and adjacent forest in Eastern DRC. International Journal of Primatology, 19 : 2007-237.

Heinrich S, Wittman TA, Ross JV, Shepherd CR, Challender DWS, Cassey P. 2017. The Global Trafficking of Pangolins: A Comprehensive Summary of Seizures and Trafficking Routes from 2010-2015. TRAFFIC, Southeast Asia Regional Office, Petaling Jaya, Selangor, Malaysia.
ICCN-DG. 2009. Plan Général de Gestion du Parc National de Kahuzi-Biega 20092019.

Ichu IG, Kambale NJ, Mousset MCL. 2017. Testing the efficacy of field surveys and local knowledge for assessing the status and threats to three species of pangolins in Cameroon, rapport de terrain, 43P.

Ingram DJ, Coad L, Abernethy KA, Maisels F, Stokes EJ, Bobo KS, Breuer T, Gandiwa E, Ghiurghi A, Greengrass E, Holmern T, Towa OWK, Obliang NAM, Poulsen RJ, Schleicher J, Nielsen MR, Solly H, Vath CL, Waltert M, Whitham ELC, Wilkie SD, Scharlemann PWJ. 2019. Assessing Africa-wide pangolin exploitation by scaling local data. Wiley Periodicals, Inc., 11: 1-9. DOI : https://doi.org/10.1111/conl.12389.

Jaramogi P. 2017. End of The Road for Kingpin Smuggling as UWA nets 6 tons of smuggled Pangolin in Tanzania. Retrieved from Online News Daily; The Investigator website http://www.theinvestigatornews.com/201 7/01/end-road-kingpin-smuggling-uwanets-6-tons-smuggled-pangolin-tanzania/ on January 23, 2017.

Kambale NJ. 2015. La consommation de la viande des brousses à Yangambi (Province de la Tshopo, RD Congo), Mémoire de Master indédit, Fac. Sci., UNIKIS, 57p.

Karawita H, Perera P, Gunawardane P, Dayawansa N. 2018. Habitat preference and den characterization of Indian Pangolin (Manis crassicaudata) in a tropical lowland forested landscape of southwest Sri Lanka, PLoS ONE, 13(11): e0206082.

DOI : https://doi.org/10.1371/journal.pone.020 6082.

Laurance WF, Croes BM, Tchignoumba L, Lahm SA, Alonso A, Lee ME, Ondzeano C. 2006. Impacts of Roads and Hunting on Central African Rainforest Mammals. Conservation Biology, 1-11. DOI : https://doi.org/10.1111/j.15231739.2006.00420.

Mambeya M, Baker F, Koumba F, Momboua BR, Hega M, Joseph V, Abernethy K. 2018. The emergence of a commercial 
trade in pangolins from Gabon. African Journal of Ecology, 601-609. DOI : https://doi.org/10.1111/aje.12507.

Mpamu BH. 2010. Etude de la filière de commercialisation de la viande de brousse à Kinshasa, mémoire inédit, Université de Kinshasa, www.memoireonline.com.

Pietersen D, Waterman C, Hywood L, Rankin P, Soewu D. 2014. Smutsia temminckii. The IUCN Red List of Threatened Species. Version 2014.3. www.iucnredlist.org.

Plumptre AJ, Kujirakwinja D, Matunguru J, Kahindo C, Kaleme P, Marks B, Muhndorfe M. 2008. Biodiversity surveys in the Misotshi-Kabogo and Marungu regions of eastern Democratic Republic of Congo. Albertine Rift Technical Reports, Series 5:1-79pp.

Robinson JG, Redford KH. 1994. Measuring the sustainability of hunting in tropical forests. Oryx28, 249-256.Steel E. A., 1994. Étude sur le volume et la valeur du commerce de la viande de brousse au Gabon. Libreville, Gabon, ministère des Eaux et Forêts et de l'Environnement, WWF, $84 \mathrm{p}$.

Soewu DA, Ayodele IA. 2009. Utilisation of Pangolin (Manis spp.) in traditional Yorubic medicine in Ijebu province, Ogun State, Nigeria. Journal of Ethnobiology and Ethnomedicine, 5: 39. DOI : https://doi.org/10.1186/17464269-5-39.

Spira C, Mitamba G, Kirkby A, Katembo J, Kiyani KC, Musikami P, Pazo, Dumbo P, Byaombe DD, Plumptre AJ, Maisels
F. 2018. Inventaire de la biodiversite dans le Parc National de Kahuzi-Biega République démocratique du Congo, rapport de terrain WCS, ICCN et CRSNLwiro, Mai, 93P.

Trageser SJ, Ghose A, Faisal M, Mro P, Mro P, Rahman CS. 2017. Pangolin distribution and conservation status in Bangladesh. PloS One, 12(4): e0175450. DOI : https://doi.org/10.1371/.0175450.

UICN. 2010. Les Parcs et réserves de la République Démocratique du Congo: Evaluation de l'efficacité de la gestion des aires protégées; Aires Protégées RDC, pdf, $149 \mathrm{P}$.

UICN. 2019. The IUCN red list of threatened species. Retrieved from http://www.iucnredlist.org/.

Waterman C, Pietersen D, Hywood L, Rankin P, Soewu D. 2014c. Smutsia gigantea. The IUCN Red List of Threatened Species 2014: e.T12762A45222061. DOI :http://dx.doi.org/10.2305/IUCN.U K.2014-2.RLTS.T12762A45222061.

Waterman C, Pietersen D, Soewu D, Hywood L, Rankin P. 2014a. Phataginus tetradactyla. The IUCN Red List of Threatened Species 2014, e.T12766A45222929. DOI : http://dx.doi.org/10.2305/IUCN.UK.201 4-2.RLTS.T12766A45222929.

Waterman C, Pietersen D, Soewu D, Hywood L, Rankin P. 2014b. Phataginus tricuspis. The IUCN Red List of Threatened Species 2014: e.T12767A45223135. DOI : http://dx.doi.org/10.2305/IUCN.UK.201 4-2.RLTS.T12767A45223135. 